\title{
Spatial characteristics of men who have sex with men and transgender women attending HIV voluntary counselling and testing in Bangkok, Thailand, 2005-2015
}

\author{
Kevin M. Weiss, ${ }^{1}$ Samart Karuchit, ${ }^{2}$ Sarika Pattanasin,,${ }^{2,4}$ Anupong Chitwarakorn, ${ }^{3}$ \\ Wipas Wimonsate, ${ }^{2,4}$ Jirawat Suksamosorn, ${ }^{2,4}$ Prin Visavakum, ${ }^{2,5}$ Wichuda Sukwicha, ${ }^{2,4}$ \\ Chaiwat Ungsedhapand, ${ }^{2,4}$ Eileen F. Dunne, ${ }^{2,4}$ Timothy H. Holtz ${ }^{2,4}$
}

\author{
${ }^{1}$ Rollins School of Public Health, Emory University, Atlanta, GA, USA; ${ }^{2}$ Thailand Ministry of Public Health \\ - U.S. Centers for Disease Control and Prevention Collaboration, Nonthaburi, Thailand; ${ }^{3}$ Department of \\ Disease Control, Thailand Ministry of Public Health, Nonthaburi, Thailand; ${ }^{4}$ Division of HIV/AIDS \\ Prevention, U.S. Centers for Disease Control and Prevention, Atlanta, Georgia, USA; ${ }^{5}$ Division of Global \\ HIV and TB, U.S. Centers for Disease Control and Prevention, Atlanta, Georgia, USA
}

\begin{abstract}
Spatiotemporal analyses can support Human Immuno-deficiency Virus (HIV) prevention programmes by identifying locations of at-risk populations in space and time, and their proximity
\end{abstract}

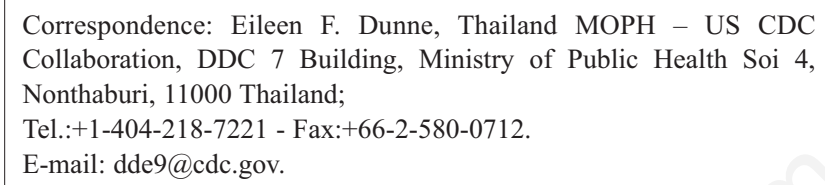

Key words: HIV; HIV testing; MSM; Thailand; Spatial analysis.

Acknowledgements: The authors kindly acknowledge and thank the personnel of the Thailand Ministry of Public Health-U.S. Centers for Disease Control and Prevention Collaboration (TUC), the Silom Community Clinic (Silom Community Clinic@TropMed), the Department of Disease Control, Thailand Ministry of Public Health, the Rainbow Sky Association of Thailand (RSAT), and the Service Workers in Group Foundation (SWING) for their support.

Meetings: Conference on Retroviruses and Opportunistic Infections, Boston, MA, Feb. 22-25, 2016.

Conflicts of Interest: The authors declare no potential conflicts of interest. CDC Disclaimer: The findings and conclusions reported in this paper are those of the authors and do not represent the views of the U.S. Centers for Disease Control and Prevention.

Received for publication: 20 May 2019.

Accepted for publication: 6 March 2020.

(C) Copyright: the Author(s), 2020

Licensee PAGEPress, Italy

Geospatial Health 2020; 15:790

doi:10.4081/gh.2020.790

This article is distributed under the terms of the Creative Commons Attribution Noncommercial License (CC BY-NC 4.0) which permits any noncommercial use, distribution, and reproduction in any medium, provided the original author(s) and source are credited. to HIV testing and prevention services. We assessed residential proximity to HIV testing venues for Men who have Sex with Men (MSM) and Transgender Women (TGW) attending Voluntary Counselling and Testing (VCT) at a large urban MSM clinic in Bangkok, Thailand in the period 2005-2015. We mapped clientprovided spatial data and HIV testing venues, calculating distance from residence to venues for VCT clients between i) September 2005-December 2009; ii) January 2010-September 2013; and iii) October 2013-May 2015. We assessed spatial characteristics across times, evaluating autocorrelation of HIV prevalence and visit density using Moran's I. Among 8,758 first-time VCT clients reporting geographic information from 2005-2015 (by period: $2737,3917,2104), 1329(15.2 \%)$ lived in postal codes $\leq 5 \mathrm{~km}$ from the clinic. Over time, the proportion living in areas covered by Bangkok postal codes $\leq 2 \mathrm{~km}$ from any MSM HIV testing venue increased from $12.6 \%$ to $41.0 \%(p<0.01)$. The proportion living $\leq 5 \mathrm{~km}$ from the clinic decreased from $16.6 \%$ to $13.0 \%(p<0.01)$. HIV prevalence and clinic visit density demonstrated statistically significant non-random spatial patterning. Significant non-random patterning of prevalent infection and client visits highlighted Bangkok's urban HIV epidemic, clinic proximity to clients, and geographic reach. Clients lived closer to testing venues, yet farther from the urban MSM clinic, over time. Spatiotemporal characteristics of VCT clients can help assess service accessibility and guide targeted prevention planning.

\section{Introduction}

Men who have Sex with Men (MSM), and Transgender Women (TGW), have a high prevalence of Human Immunodeficiency Virus (HIV) in many global settings, including Southeast Asia (Beyrer et al., 2012). In Thailand's concentrated epidemic, the prevalence of HIV among MSM (9.1\% in 2018; UNAIDS, 2018) is higher than the general population, and new HIV infections are primarily occurring in young MSM and TGW (van Griensven et al., 2013; Sullivan et al., 2015). HIV testing is a core platform for entry into HIV prevention, through strategies like Pre-Exposure Prophylaxis (PrEP) and Treatment as Prevention (TasP), including efforts to reduce viral loads to undetectable and untransmissible levels (WHO, 2014; Guadamuz et 
al., 2015). Assessments of access to HIV testing in key populations using geospatial data provide information on whom is being served and where, as well as potential gaps in service delivery.

Spatial methods, such as the use of Geographic Information Systems (GIS), can be used to assess spatial patterns, movement, and trends in populations and identify geographic correlates that may impact health behaviours and service utilization, including HIV testing, diagnosis, and infection acquisition (Anselin et al., 2010; Yao et al., 2014; Hixson et al., 2011). Published spatial analyses of HIV in Thailand have been limited, with most occurring in the 1990s; those analyses focused on the heterosexual epidemic and included descriptions of the distribution of support organizations, HIV seroprevalence and its correlates, and commercial sex work (Torugsa et al., 2003; Sirisopana et al., 1996; Del Casino, 2001; Jongsthapongpanth et al., 2010). To our knowledge, few spatial analyses of HIV have focused on Bangkok or explicitly examined the geographic distribution of testing venues, individuals seeking HIV testing, or the HIV epidemic among Thai MSM and TGW.

The Silom Community Clinic (SCC), an anonymous, gay and transgender-friendly clinic in Bangkok, Thailand, providing voluntary counselling and testing (VCT) for HIV and sexually transmitted infection (STI) services, was established in 2005 by the Thailand Ministry of Public Health (MOPH) - U.S. Centers for Disease Control and Prevention (CDC) Collaboration (TUC) initially with location at the Bangkok Christian Hospital and later at the Mahidol University Hospital for Tropical Diseases (van Griensven et al., 2013; Holtz et al., 2012).

We aimed to assess spatial and demographic characteristics of first-time SCC clients over time to describe changes in our client population and accessibility to HIV testing venues from 2005 to 2015. Spatial patterns of clients could impact testing and prevention service utilization and may help support the design and location of future prevention programs and activities.

\section{Materials and Methods}

\section{Demographic Characteristics and HIV Testing Methods}

We collected demographic and spatial information from gay, bisexual, and other MSM and TGW attending SCC for VCT from September 2005-May 2015. Postal code of residence at first visit and postal code of birth were routinely self-reported during VCT at visit registration. Participants reporting incomplete geographic data and repeat visits by the same participant were excluded from this analysis. Clients were screened for HIV-1 and HIV-2, adhering to Thai national guidelines by using a series of three rapid diagnostic tests (Determine, Abbott Laboratories, Abbott Park, IL, USA; DoubleCheck, Orgenics, Yavne, Israel, or SD Bioline, Standard Diagnostics, Kyonggi-do, South Korea; and Capillus HIV-1/2, Trinity Biotech, Carlsbad, CA, USA or HIV1/2 Core, Core Diagnostic, Birmingham, UK) (van Griensven et al., 2013; van Griensven et al., 2015). Beginning in 2009, we performed additional Nucleic Acid Amplification Testing (NAAT) (Aptima Genprobe, Gen-Probe, San Diego, CA, USA) and fourth generation immunoassays (AxSYM-HIV Ag/AB, Abbott Laboratories or Cobas Core, Core HIV Combi, Roche Diagnostics, Mannheim, Germany) for detection of acute HIV infection in clients who had a negative rapid HIV test result.
Data from the initial VCT visit for first-time unique individuals were described in three time frames, reflecting different periods of MSM service delivery: 1) September 1, 2005-December 31, 2009, SCC's initial years and when there was only a small number of MSM-focused venues, 2) January 1, 2010-September 30, 2013, a time frame in which the number of MSM venues nearly tripled and 3) October 1, 2013-May 31, 2015, after SCC moved to SCC (a)TropMed and MSM venues continued to increase in number.

\section{Geographic Methods}

Geographic shapefiles of postal codes in Thailand and the 50 districts within the city of Bangkok (Bangkok) were obtained from CDC and the Thailand Department of Provincial Administration (Samart Karuchit, personal communication). City districts could be composed of multiple postal codes, or a postal code could be comprised of areas in multiple districts. The median size of the postal codes in the city of Bangkok was $31.3 \mathrm{~km}^{2}$ (mean $=63.9$ $\mathrm{km}^{2}$ ), while the postal codes outside the city were generally larger due to the truncation down to 2 digits (e.g., 97100 and 97200 both become 97000). These shapefiles were used to further categorize reported participant residence and birth postal codes as either: 1) in the city of Bangkok (referred to as Bangkok), 2) in the city's five neighbouring provinces, Nonthaburi, Nakhom Pathom, Samut Sakhon, Pathum Thani and Samut Prakan (referred to as neighbouring provinces in the Greater Bangkok Metropolitan Area [BMA]), or 3) other provinces in Thailand outside the Greater BMA area (all referred to as 'other Thailand provinces'). Postal code locations within Bangkok were further stratified by location $\leq 2 \mathrm{~km}$ from any public MSM HIV testing venue.

Change in location since birth was determined by comparing reported postal codes of birth and residence. Participants were classified as having: 1) moved from outside Bangkok into the city, 2) moved out of the city of Bangkok, 3) remained in the same area (e.g., having been born in the city and reporting a current residence in the city), or 4) followed another pattern (e.g. moving from one of the five surrounding provinces in the Greater BMA to a different area of Thailand). We obtained a list of public HIV testing venues for MSM over the study period (personal communication, Prin Visavakum), and these locations were geocoded and exported to ArcGIS 10.2 (ESRI, Redlands, CA, USA).

We used postal code centroids to assess distance from the participant's postal code of residence to time-concordant venues. Polygon-based buffers were created encompassing two $\mathrm{km}$ and five $\mathrm{km}$ radii around testing venues. Clients living in postal codes which contained testing venues, or whose centroids were captured in these buffers, were respectively classified under two proximity schemes: living within $2 \mathrm{~km}$ of any testing venue or living within $5 \mathrm{~km}$ of any testing venue. GIS analysis of spatial autocorrelation measures for postal code visit density (percentage of included client visits contributed by a postal code) and HIV prevalence density (postal code client HIV prevalence). This approach used nearest neighbour weighting to create a spatial weights matrix. Choropleth maps of client residential postal code and prevalent HIV infection at first visit were created and overlaid with HIV testing venue locations.

The provision of VCT services was determined to be a public health program activity by the Office of the Associate Director of Science, U.S. CDC and, as such, this analysis of public health service delivery was exempt from CDC IRB review. This activity was funded by the CDC. 


\section{Statistical Analysis Methods}

Demographic and spatial characteristics were described across time frames using proportions, means, and medians, with betweentime frame characteristic differences assessed using CochranArmitage tests of trend for binary variables and chi-square tests of association for categorical variables. Bivariate associations between factors and prevalent, non-NAAT-positive HIV infection are reported as crude prevalence Odds Ratios (cORs) with $95 \%$ Confidence Intervals (CIs). Covariates associated at a $p<0.10$ significance level were entered into a multivariable logistic regression model. Final model results, reported as adjusted prevalence Odds Ratios (aOR) with associated 95\% CIs, included variables significant at a $p<0.05$ level after backwards elimination and assessments of collinearity and two-way interactions. Data analysis was performed in SAS 9.4 (Cary, NC, USA). Global Moran's I tests for spatial dependency (autocorrelation) and clustering for visit density and HIV prevalence density were performed in ArcGIS.

\section{Results}

From September 2005 to May 2015, 8,945 unique VCT clients visited SCC, with 8,758 (97.9\%) reporting complete geographic information. Among clients with geographic information, 2,737 (31.3\%) visited during the first-time frame (Sept. 2005-Dec. 2009), 3,917 (44.7\%) visited during the second time frame (Jan. 2010Sept. 2013), and 2104 (24.0\%) visited during the third time frame (Oct. 2013-May 2015) (Table 1). A majority (64.2\%) were aged between 18-29 years and 2,342 (31.1\%) of those with HIV test results were HIV-seropositive (excluding acute infection) at first visit, ranging from $25.8 \%$ in the first-time frame to $33.7 \%$ and $32.6 \%$ in the latter two-time frames. Only half of first-time VCT clients had previously been tested for HIV and only $3.1 \%$ did not identify as MSM (data not shown). Around three-quarters (76.3\%) of the clients resided in a Bangkok postal code, with 2677 (30.6\%) clients living in a Bangkok postal code $\leq 2 \mathrm{~km}$ from any public HIV testing venue during the time frame of their visit. About one in six clients (15.2\%) lived in postal codes with centroids located $\leq 5 \mathrm{~km}$ from SCC, while nearly half (46.0\%) had moved to Bangkok since birth. Postal code visit density, i.e. the percentage of visits contributed by residents of a postal code, is shown for the entire study time frame in Figure 1, with time frame-specific visit densities represented in Figure 2. Four HIV testing venues existed during the first-time frame, increasing to 23 venues in later time frames. An increasing proportion of clients lived in postal codes $\leq 2 \mathrm{~km}$ from any testing venue $(12.6 \%, 37.5 \%, 41.0 \%, p<0.01)$, and a decreasing proportion of clients lived in postal codes $\leq 5 \mathrm{~km}$ of the SCC

Table 1. Socio-demographic and behavioural factors by time frame of first VCT visit for 8,758 clients attending Silom Community Clinic and reporting valid geographic information (2005-2015).

\begin{tabular}{|c|c|c|c|c|c|}
\hline Respondent characteristics & $\begin{array}{l}\text { Sep } 2005-D e c 2009 \\
(N=2,737) \\
N(\%)\end{array}$ & $\begin{array}{c}\operatorname{Jan} 2010-\operatorname{Sep} 2013 \\
(\mathrm{~N}=3,917) \\
\mathrm{N}(\%)\end{array}$ & $\begin{array}{l}\text { Oct 2013-May } 2015 \\
(\mathrm{~N}=2,104) \\
\mathrm{N}(\%)\end{array}$ & $\begin{array}{l}\text { Total } \\
(\mathrm{N}=8,758) \\
\mathrm{N}(\%)\end{array}$ & p value** \\
\hline $\begin{array}{l}\text { Age at entry (years) } \\
<18 \\
18-24 \\
25-29 \\
\geq 30\end{array}$ & $\begin{array}{l}40(1.5) \\
921(33.6) \\
829(30.3) \\
947(34.6)\end{array}$ & $\begin{array}{c}40(1.0) \\
1,205(30.8) \\
1,156(29.5) \\
1,516(38.7)\end{array}$ & $\begin{array}{l}29(1.4) \\
807(38.4) \\
593(28.2) \\
675(32.1)\end{array}$ & $\begin{array}{c}109(1.2) \\
2,933(33.5) \\
2,578(29.4) \\
3,138(35.8)\end{array}$ & $<0.01^{\mathrm{a}}$ \\
\hline $\begin{array}{l}\text { Human immunodeficiency virus infection }{ }^{\mathrm{c}} \\
\text { Antibody-positive }\end{array}$ & $554(25.8)$ & $1,149(33.7)$ & $639(32.6)$ & $2,342(31.1)$ & $<0.01^{\mathrm{b}}$ \\
\hline $\begin{array}{l}\text { Postal code of birthplace }^{\mathrm{d}} \\
\text { Bangkok } \\
\text { Neighbouring provinces in Greater BMA } \\
\text { Other provinces in Thailand }\end{array}$ & $\begin{array}{c}896(37.4) \\
144(6.0) \\
1,356(56.6)\end{array}$ & $\begin{array}{l}1,347(34.6) \\
266(6.8) \\
2,278(58.5)\end{array}$ & $\begin{array}{c}681(32.4) \\
166(7.9) \\
1,255(59.7)\end{array}$ & $\begin{array}{c}2,924(34.9) \\
576(6.9) \\
4,889(58.3)\end{array}$ & $<0.01^{\mathrm{a}}$ \\
\hline $\begin{array}{l}\text { Distance from residence at first visit to any testing } \\
\text { Bangkok postal code } \leq 2 \mathrm{~km} \text { from testing venue } \\
\text { Bangkok postal code }>2 \mathrm{~km} \text { from testing venue } \\
\text { Neighboring provinces in Greater BMA } \\
\text { All other Thailand provinces }\end{array}$ & $\begin{array}{c}344(12.6) \\
1,805(65.9) \\
353(12.9) \\
235(8.6)\end{array}$ & $\begin{array}{c}1,470(37.5) \\
1,541(39.3) \\
577(14.7) \\
329(8.4)\end{array}$ & $\begin{array}{l}863(41.0) \\
657(31.2) \\
379(18.0) \\
205(9.7)\end{array}$ & $\begin{array}{c}2,677(30.6) \\
4,003(45.7) \\
1,309(14.9) \\
769(8.8)\end{array}$ & $<0.01^{\mathrm{a}}$ \\
\hline $\begin{array}{l}\text { Distance to SCC location at first visit } \\
\text { Postal code residence } \leq 2 \mathrm{~km} \text { of SCC location } \\
\text { Postal code residence } \leq 5 \mathrm{~km} \text { of SCC location }\end{array}$ & $\begin{array}{c}132(4.8) \\
454(16.6)\end{array}$ & $\begin{array}{c}139(3.5) \\
601(15.3)\end{array}$ & $\begin{array}{c}185(8.8) \\
274(13.0)\end{array}$ & $\begin{array}{c}456(5.2) \\
1,329(15.2)\end{array}$ & $\begin{array}{l}<0.01^{\mathrm{b}} \\
<0.01^{\mathrm{b}}\end{array}$ \\
\hline $\begin{array}{l}\text { Location since birth }^{\mathrm{d}} \\
\text { Change from outside Bangkok to Bangkok } \\
\text { Change from Bangkok to outside Bangkok } \\
\text { Stayed in same geographic area classification } \\
\text { Other movement pattern }\end{array}$ & $\begin{array}{l}1,076(44.9) \\
98(4.1) \\
1,093(45.6) \\
129(5.4)\end{array}$ & $\begin{array}{l}1,832(47.1) \\
188(4.8) \\
1,641(42.2) \\
230(5.9)\end{array}$ & $\begin{array}{l}948(45.1) \\
111(5.3) \\
861(41.0) \\
182(8.7)\end{array}$ & $\begin{array}{l}3,856(46.0) \\
397(4.7) \\
3,595(42.9) \\
541(6.4)\end{array}$ & $<0.01^{\mathrm{a}}$ \\
\hline
\end{tabular}

* Individual categories sum down columns, total column sums values across rows; ${ }^{* *}$ p value is association between time frame and characteristic, using either Chi square test of association or Cochran-Armitage Trend test; ${ }^{+}$Bangkok postal codes > two km from a testing venue exclude venues located in neighboring provinces in the Greater BMA; ${ }^{++}$Greater BMA $=$Greater Bangkok Metropolitan Area (Includes the city of Bangkok

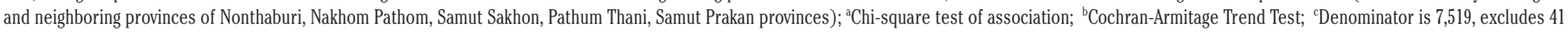
individuals with NAAT-positive and serology-negative test results and 1,198 individuals without antibody testing results; ${ }^{\circledR} 369$ missing values; Bangkok = City of Bangkok; VCT = Voluntary Counselling and Testing; SCC = Silom Community Clinic. 


\section{Article}

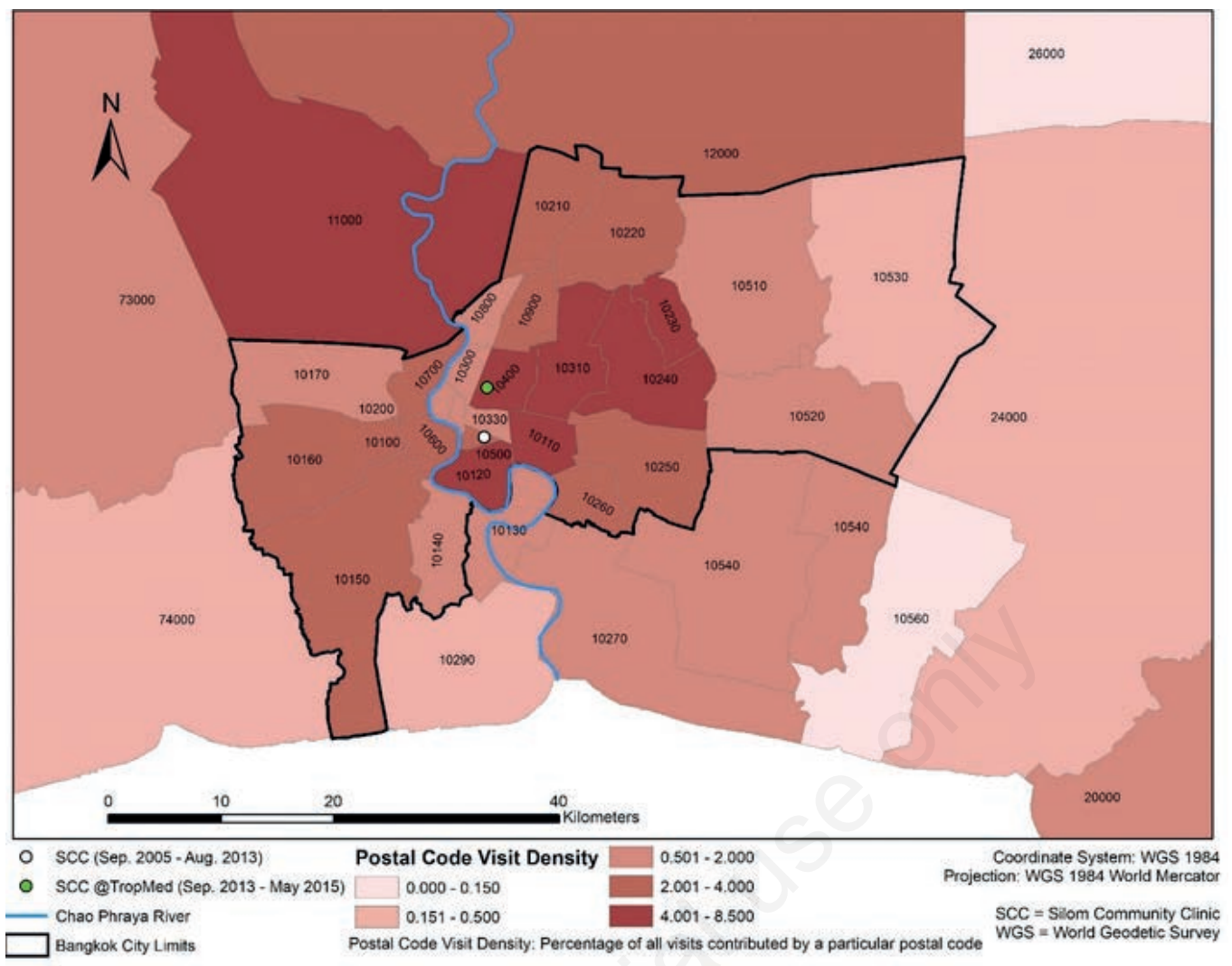

Figure 1. Downtown Bangkok postal codes covered by SCC, 2005-2015.
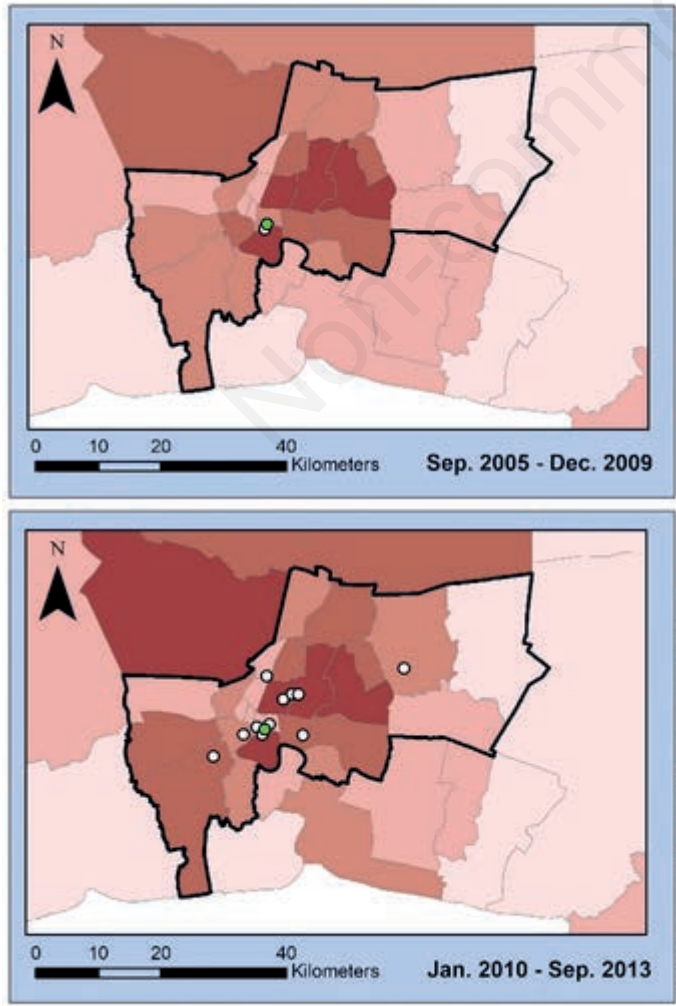

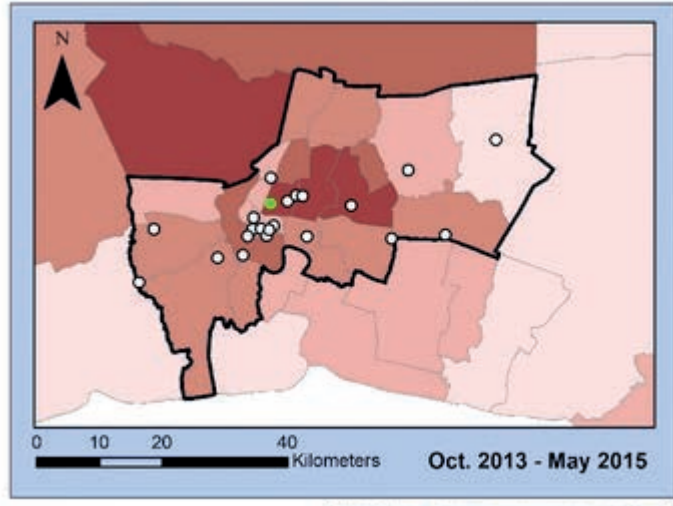

- SCC Venues

- Public MSM HIV Testing Venues Bangkok City Limits

Postal Code Period Visit Density $0.00-0.50$

$0.51-1.50$

$1.51-3.00$

$3.01-5.00$

$5.01-9.00$

Coordinate System: WGS 1984 Projection: WGS 1984 World Mercator $\mathrm{HIV}=$ Human immunodeficiency virus MSM $=$ Men who have sex with men SCC $=$ Silom Community Clinic WGS $=$ World Geodetic Survey

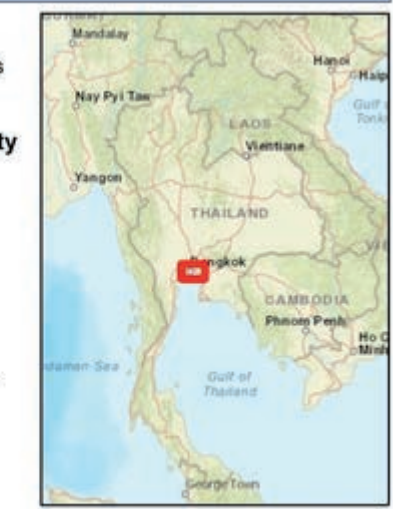

Period Visit Density: Percentage of all visits in a given time period contributed by a particular postal code

Figure 2. Increasing numbers of HIV testing venues for Thai MSM, but similar client distribution, during three time frames. 
location open during that time frame $(16.6 \%, 15.3 \%, 13.0 \%$, $p<0.01)$.

Tests of spatial autocorrelation for both 1) postal code visit density, and 2) prevalent HIV infection density were significant by the Global Moran's $I$ statistic $(I=0.23$ and $p<0.01, I=0.19$ and $p<0.01$, respectively). Birth in a Bangkok postal code $(\mathrm{cOR}=0.64$, $95 \%$ CI, 0.58-0.72), current residence in a postal code in Bangkok $\leq 5 \mathrm{~km}$ from an SCC location $(\mathrm{cOR}=0.81,95 \% \mathrm{CI}, 0.70-0.93)$ and age below $18(\mathrm{cOR}=0.48,95 \% \mathrm{CI}, 0.28-0.84)$ were all significantly associated with reduced odds of prevalent HIV infection in bivariate analyses (Table 1). Additionally, a first visit to SCC in a later time frame (Jan 2010-Sep $2013 \mathrm{cOR}=1.47,95 \%$ CI, 1.301.65; Oct 2013-May 2015 cOR = 1.40, 95\% CI, 1.22-1.60), movement into Bangkok $(\mathrm{cOR}=1.53,95 \% \mathrm{CI}, 1.38-1.70)$ and age greater than $18-24$ years (aged $25-29$ years cOR $=1.33,95 \% \mathrm{CI}$, $1.18-1.50 ; \geq 30$ years cOR $=1.35,95 \%$ CI, $1.20-1.52$ ) were significantly associated with increased odds of prevalent HIV infection in bivariate analyses.

In multivariable logistic regression analysis, movement into Bangkok $(\mathrm{aOR}=1.52,95 \% \mathrm{CI}, 1.37-1.69)$ was significantly associated with increased odds of prevalent HIV infection. HIV seropositive infection was less likely among clients living $\leq 5 \mathrm{~km}$ from SCC ( $\mathrm{aOR}=0.80,95 \% \mathrm{CI}, 0.69-0.92)$ (Table 2). More recent clients had greater odds of being HIV seropositive (Jan 2010-Sep $2013 \mathrm{aOR}=1.37,95 \%$ CI, 1.21-1.55; Oct. 2013-May $2015 \mathrm{aOR}$ $=1.33,95 \% \mathrm{CI}, 1.16-1.53)$. Increased age was also associated with increased odds of prevalent infection (25-29 $\mathrm{cOR}=1.34$, $95 \%$ CI, $1.18-1.52 ; \geq 30 \mathrm{cOR}=1.39,95 \%$ CI, 1.23-1.57). Postal code at birth was removed from the multivariable model due to issues of collinearity with postal code of residence. No two-way interaction terms were significant.

\section{Discussion}

This analysis describes spatiotemporal characteristics of Thai MSM and TGW attending SCC over a 10-year period. We found that first-time clients had varied spatial characteristics over time, with evidence that clients lived closer to HIV testing and prevention services. Overall, we see this as a positive trend for access to services for these key populations. Geographic attributes, including movement and distance to HIV testing venues, were significant predictors of prevalent HIV infection, and there was significant spatial patterning of residential locations of all and HIV-positive VCT clients. Describing spatiotemporal characteristics of populations at risk for HIV can support tailored HIV prevention interventions. An increased number of testing sites may not result in increased testing among Thai MSM, however, as factors such as risk perception and venue environment might affect testing uptake.

This analysis expands on previous Thai HIV spatial research, yet is the first to focus on the key populations of MSM and TGW. A previous study of more than 400,000 Royal Thai Army recruits demonstrated a wide geographic distribution of the HIV epidemic (Torugsa et al., 2003). Geographic correlates, namely residence in a high-seroprevalence region (Sirisopana et al., 1996) and commercial sex work concentration (Jongsthapongpanth et al., 2010) were associated with prevalent HIV infection. These assessments in Thailand primarily occurred in the 1990s-2000s and focused on the heterosexual HIV epidemic. Since this time, there has been a shift of the HIV epidemic to key populations of MSM and TGW (National AIDS Committee, 2015).

To date, there have been no spatiotemporal assessments of MSM and TGW in Thailand, and this descriptive analysis could serve as a foundation for more advanced or targeted research. As

Table 2. Crude and adjusted prevalence odds ratios and 95\% confidence intervals for correlates of being HIV seropositive at first VCT visit, SCC (2005-2015). (Adjusted Model N=7,228).

\begin{tabular}{|c|c|c|}
\hline \multirow[t]{2}{*}{ Characteristic } & \multicolumn{2}{|c|}{ Prevalent HIV infection } \\
\hline & Crude Odds Ratio (95\% CI) & Adjusted Odds Ratio (95\% CI) \\
\hline \multicolumn{3}{|l|}{ Age at entry (years) } \\
\hline$<18$ & $0.48(0.28-0.84)$ & $0.53(0.30-0.94)$ \\
\hline $18-24$ & Referent & Referent \\
\hline $25-29$ & $1.33(1.18-1.50)$ & $1.34(1.18-1.52)$ \\
\hline$\geq 30$ & $1.35(1.20-1.52)$ & $1.39(1.23-1.57)$ \\
\hline \multicolumn{3}{|l|}{ Postal code of birthplace } \\
\hline Bangkok & $0.64(0.58-0.72)$ & Removed for Collinearity \\
\hline Neighboring provinces in Greater BMA & $0.83(0.68-1.02)$ & Removed for Collinearity \\
\hline Other provinces in Thailand & Referent & Removed for Collinearity \\
\hline Postal code residence $\leq 5 \mathrm{~km}$ of SCC location & $0.81(0.70-0.93)$ & $0.80(0.69-0.92)$ \\
\hline \multicolumn{3}{|l|}{ Time frame of first visit } \\
\hline Sep 2005-Dec 2009 & Referent & Referent \\
\hline Jan 2010-Sep 2013 & $1.47(1.30-1.65)$ & $1.37(1.21-1.55)$ \\
\hline Oct 2013-May 2015 & $1.40(1.22-1.60)$ & $1.33(1.16-1.53)$ \\
\hline \multicolumn{3}{|l|}{ Location since birth } \\
\hline Change from outside Bangkok to Bangkok & $1.53(1.38-1.70)$ & $1.52(1.37-1.69)$ \\
\hline Change from Bangkok to outside Bangkok & $1.11(0.87-1.42)$ & $1.08(0.85-1.38)$ \\
\hline Stayed in same geographic area classification & Referent & Referent \\
\hline Other movement pattern & $1.29(1.04-1.59)$ & $1.22(0.98-1.51)$ \\
\hline
\end{tabular}

$\mathrm{Cl}$ = Confidence Interval; Bangkok = City of Bangkok; Greater BMA = Greater Bangkok Metropolitan Area (Includes the city of Bangkok and neighboring provinces of Nonthaburi, Nakhom Pathom, Samut Sakhon, Pathum Thani, Samut Prakan provinces); VCT = voluntary counseling and testing; SCC = Silom Community Clinic. 
governments and other stakeholders are working to get to no new HIV infections, geospatial assessments can identify where to target efforts and programs. Further spatial information on sexual networks, which are a key determinant of HIV risk among MSM, to inform network-level interventions can help improve service accessibility and address risk behaviours (Amirkhanian, 2014). Although not representative of all MSM and TGW, clients attending SCC VCT clinics that have served MSM for over a decade provide a large, geographically diverse MSM client population to assess service accessibility in the absence of true population-based representative samples. Routine collection of geographic information on all HIV VCT clients in Bangkok could further this understanding. Our study found that an increasing proportion of firsttime clients attending SCC lived $\leq 2 \mathrm{~km}$ from any public MSM testing venue over time, rising significantly from $12.6 \%$ to $41.0 \%$ by the last time frames. Two $\mathrm{km}$ represented a reasonable walking distance for clients and staff, and five $\mathrm{km}$ represented a reasonable motorbike or taxi distance. This suggests that HIV testing venues for MSM and TGW seeking VCT have become more accessible over time in Bangkok, Thailand. The community recognized the MSM/TGW HIV epidemic in the early 2000s, and, subsequently, Thailand has made efforts to increase service delivery to MSM/TGW, including geographic and numeric expansion of MSM-friendly HIV testing. These vary in environment, cost to the client, operating hours, and types of services provided, but this is a positive step in addressing the HIV epidemic in Thailand.

Accessibility to HIV testing is a key factor that can influence the likelihood of being tested for HIV; more testing venues and sites, and an increase of self-testing and mobile testing efforts, can boost testing rates among those at risk (Yao et al., 2014; KhumaloSakutukwa et al., 2008; Kawichai et al., 2012). An Atlanta-based analysis of linkage to HIV care and viral suppression highlighted the interaction between where one lives and social factors, such as poverty, that can drive access issues potentially leading to onward HIV transmission (Goswami et al., 2016). Greater access to testing for key populations can enable utilization of HIV-related services, supporting both HIV prevention and HIV care and treatment (WHO, 2014). Service delivery, including hours of operation, cost, environment, ease of use, and types of services offered, can also influence and support HIV testing and HIV prevention efforts (Kawichai et al., 2012). Evaluation of location, and proximity of HIV testing venues to MSM and TGW, can support optimal HIV testing services.

Although there was an increase in the number of first-time MSM client visits at SCC during the study's entire time frame, other settings in Bangkok did not meet HIV testing targets for MSM and other at-risk key populations (National AIDS Committee, 2015). MSM face potential stigma, violence, discrimination, and criminalization, which are associated with fear of seeking care and lower rates of service utilization (Baral et al., 2014). A study of UN country reports showed that only about a third of Thai MSM had been tested for HIV, while only a quarter of South and Southeast Asian MSM were reached by HIV prevention programs (Adam et al., 2009). Expanding access to care, beginning with testing, and convenience of care locations could contribute to better engagement in care. In addition, venues need to consider their service delivery model for key populations to ensure protection and safety and ease of use and comfort. More efforts may be needed to consider a centre of excellence model for service delivery to key populations in Southeast Asia.

Spatial analysis can describe geographic variation and cluster- ing, as well as risk factors, of infectious diseases, including HIV (Wand et al., 2015; Zulu et al., 2014). Recent work in China has described the county-level distribution of HIV cases, analysing the national case registry through spatial regression to detect disease clustering and correlates of prevalence (Qin et al., 2017). Our study found there was significant global autocorrelation, or nonrandom patterning, for prevalent HIV infection and client visit density, manifesting as, respectively, neighbouring postal codes with similar prevalence of HIV-positive VCT clients and overall VCT clients. This finding reflects the urban location of both SCC clinics as well as the urban nature of the HIV epidemic in Thai MSM (van Griensven et al., 2013; Sullivan et al., 2015).

Notably, only $15 \%$ of SCC clients came from postal codes within $5 \mathrm{~km}$, highlighting the wide geographic reach of a longestablished clinic. This may indicate that SCC model for service delivery, including evening operating hours, well-trained staff, focus on confidentiality and client protections, engagement with the community, and no-cost services are attracting MSM from outside their immediate residential areas. Loyalty to this clinic model may translate to interest in follow-up and retention in care, augmenting the initial first visit with repeat prevention messages and opportunities. There may also be a lack of awareness of more conveniently located MSM testing venues. It is unknown whether Thai MSM living near MSM HIV testing clinics are more likely to test elsewhere as a result of potential stigma or unwanted disclosure of sexual orientation associated with visiting a nearby MSM clinic. It is also unknown whether these clients may have also received HIV testing at other closer venues. Future assessments of the location of MSM populations in Bangkok with more granular geo-mapping information, such as that provided by a mobile phone or other application, and service provision across venues could evaluate whether there may be unidentified gaps in service delivery for MSM testing.

This study has several limitations. Our assessment only evaluated first visits of clients attending SCC and this sample may not be representative of all MSM in Bangkok. Given that SCC was one of the largest, and only, HIV service providers during these time frames, it may offer insight on access to care; we are not aware of any representative samples of Bangkok MSM with geocoding data available (van Griensven et al., 2013). SCC moved to a different, urban location in downtown Bangkok at the beginning of the third time frame. Evaluation of HIV testing and access by postal code may be subject to the ecological fallacy of applying larger characteristics to an individual level, as postal codes may not necessarily reflect underlying population distribution, and different spatial units could lead to different conclusions (Piantadosi et al., 1988; Grubesic et al., 2006). Further integration of other measures of service access and utilization, such as the collection of more granular location data or reasons for accessing a particular service location would enhance the robustness of these findings. Additionally, centroid-based distance analysis and lack of residential point data might obscure individual-level accessibility trends; a postal code of residence may not correlate perfectly with areas where key populations are spending most of their time or exhibiting risk behaviours.

Considering the limitations of ecologic and centroid-based analyses, these spatial findings are best used for exploratory assessment and hypothesis generation, rather than as concrete explanations of individual-level risks. The collection of more local, disaggregated data, such as sub-district-level or even specific GIS coordinates of home, work, and social environments, may 
help us better understand spatial patterns beyond those seen at a postal code level. Future spatial assessments that include all MSM in Bangkok, rather than those attending a single venue, can better characterize key population locations as well as the availability of HIV prevention and care services. This could include evaluations using GIS in smartphone applications to assess questions such as the impact of geography accessibility on uptake of HIV prevention and care services to key populations. A large systematic review found that more than three-quarters of studies identified that greater distance or travel time to care was associated with worse health outcomes (Kelly et al., 2016). Recent studies have identified spatial access as a key predictor of willingness to use HIV PrEP (Ojikutu et al., 2019) and loss-to-follow-up from HIV care (Bilinski et al., 2017). Targeted prevention and care efforts and programs, including focusing on geographic hotspots, may benefit from the findings of basic and more advanced geospatial analyses using individual and aggregated data. Characterization of the demographics, location, and movement of persons at risk for HIV can help identify the best locations and methods for health services to support HIV prevention in the community. Regular collection and analysis of spatial data from HIV prevention programs, both clinic-based and mobile, is needed and can help determine whether greater proximity to HIV testing venues can improve testing rates and service utilization in Thai MSM. The use of spatial techniques and data visualization can serve to support public health programs and policies, but can also serve as a tool for effective communication to stakeholders and policymakers about the local context of the epidemic (Torugsa et al., 2003).

\section{References}

Adam PCG, de Wit JBF, Toskin I, et al., 2009. Estimating levels of HIV testing, HIV prevention coverage, HIV knowledge, and condom use among men who have sex with men (MSM) in low-income and middle-income countries. J Acquir Immune Defic Syndr 52 Suppl 2:S143-51.

Amirkhanian YA, 2014. Social networks, sexual networks and HIV risk in men who have sex with men. Curr HIV/AIDS Rep 11:81-92.

Anselin L, Syabri I, Kho Y, 2010. GeoDa: An Introduction to Spatial Data Analysis. In: Handbook of Applied Spatial Analysis. Berlin, Heidelberg: Springer Berlin Heidelberg, pp. 73-89.

Baral S, Holland CE, Shannon K, et al., 2014. Enhancing benefits or increasing harms: community responses for HIV among men who have sex with men, transgender women, female sex workers, and people who inject drugs. JAIDS J Acquir Immune Defic Syndr 66:S319-28.

Beyrer C, Baral SD, van Griensven F, Goodreau SM, Chariyalertsak S, Wirtz AL, Brookmeyer R, 2012. Global epidemiology of HIV infection in men who have sex with men. Lancet 380:367-77.

Bilinski A, Birru E, Peckarsky M, et al., 2017. Distance to care, enrollment and loss to follow-up of HIV patients during decentralization of antiretroviral therapy in Neno District, Malawi: A retrospective cohort study. Anglewicz P, ed. PLoS One 12:e0185699. doi:10.1371/journal.pone.0185699.

Del Casino VJ, 2001. Healthier geographies: Mediating the gaps between the needs of people living with HIV and AIDS and health care in Chiang Mai, Thailand. Prof Geogr 53:407-21.
Goswami N, Schmitz M, Sanchez T, et al., 2016. Understanding local spatial variation along the care continuum: The potential impact of transportation vulnerability on HIV linkage to care and viral suppression in high-poverty areas, Atlanta, Georgia. JAIDS 72:65-72.

Grubesic TH, Matisziw TC, 2006. On the use of ZIP Codes and ZIP Code Tabulation Areas (ZCTAs) for the spatial analysis of epidemiological data. Int J Health Geogr 5:58.

Guadamuz TE, Cheung DH, Wei C, Koe S, Lim SH, 2015. Young, online and in the dark: Scaling up HIV testing among MSM in ASEAN. PLoS One 10:e0126658.

Hixson BA, Omer SB, del Rio C, Frew PM, 2011. Spatial clustering of HIV prevalence in Atlanta, Georgia and population characteristics associated with case concentrations. J Urban Health 88:129-41.

Holtz TH, Thienkrua W, McNicholl JM, et al., 2012. Prevalence of Treponema pallidum seropositivity and herpes simplex virus type 2 infection in a cohort of men who have sex with men, Bangkok, Thailand, 2006-2010. Int J STD AIDS 23:424-8.

Jongsthapongpanth A, Bagchi-Sen S, 2010. The context and impact of HIV and AIDS in Chiang Rai, Thailand: A study of youth and young adults. Ann Assoc Am Geogr 100:30-56.

Kawichai S, Celentano D, Srithanaviboonchai K, et al., 2012. NIMH Project Accept (HPTN 043) HIV/AIDS community mobilization (CM) to promote mobile HIV voluntary counseling and testing (MVCT) in rural communities in Northern Thailand: Modifications by experience. AIDS Behav 16:122737.

Kelly C, Hulme C, Farragher T, Clarke G, 2016. Are differences in travel time or distance to healthcare for adults in global north countries associated with an impact on health outcomes? A systematic review. BMJ Open 6:e013059. doi:10.1136/ bmjopen-2016-013059.

Khumalo-Sakutukwa G, Morin SF, Fritz K, et al., 2008. Project Accept (HPTN 043): a community-based intervention to reduce HIV incidence in populations at risk for HIV in subSaharan Africa and Thailand. J Acquir Immune Defic Syndr 49:422-31.

National AIDS Committee, 2015. Thailand: Ending AIDS 2015: Global AIDS Response Progress Report. Available from: http://www.unaids.org/sites/default/files/country/documents/T HA_narrative_report_2015.pdf.

Ojikutu BO, Bogart LM, Mayer KH, Stopka TJ, Sullivan PS, Ransome Y, 2019. Spatial Access and Willingness to Use PreExposure Prophylaxis Among Black/African American Individuals in the United States: Cross-Sectional Survey. JMIR public Heal Surveill 5:e12405. doi:10.2196/12405.

Piantadosi S, Byar DP, Green SB, 1988. The ecological fallacy. Am J Epidemiol 127:893-904.

Qin Q, Guo W, Tang W, et al., 2017. Spatial analysis of the human immunodeficiency virus epidemic among men who have sex with men in China, 2006-2015. Clin Infect Dis 64:956-63.

Sirisopana N, Torugsa K, Mason CJ, et al., 1996. Correlates of HIV-1 seropositivity among young men in Thailand. J Acquir Immune Defic Syndr Hum Retrovirology 11:492-8.

Sullivan PS, Beyrer C, 2015. HIV in Thai men who have sex with men: a sustained emergency. Lancet HIV 2:e38-9.

Torugsa K, Anderson S, Thongsen N, et al., 2003. HIV epidemic among young Thai men, 1991-2000. Emerg Infect Dis 9:881-3.

UNAIDS, 2018. Geneva, Switzerland; 2018. Available from: http://www.unaids.org/sites/default/files/media_asset/unaids- 
data-2018 en.pdf.

van Griensven F, Holtz TH, Thienkrua W, et al., 2015. Temporal trends in HIV-1 incidence and risk behaviours in men who have sex with men in Bangkok, Thailand, 2006-13: an observational study. Lancet HIV 2:e64-70.

van Griensven F, Thienkrua W, McNicholl J, et al., 2013. Evidence of an explosive epidemic of HIV infection in a cohort of men who have sex with men in Thailand. AIDS 27:825-32.

Wand H, Ramjee G, 2015. Spatial clustering of "measured"; and "unmeasured" risk factors for HIV infections in hyper-endemic communities in KwaZulu-Natal, South Africa: results from geoadditive models. AIDS Care 27:1375-81.
WHO, 2014. Consolidated guidelines on HIV prevention, diagnosis, treatment, and care for key populations. 2014. Available from: http://apps.who.int/iris/ bitstream/10665/128048/1/ 978924150 7431_eng.pdf?ua=1\&ua=1. Accessed: June 4, 2017.

Yao J, Agadjanian V, Murray AT, 2014. Spatial and social inequities in HIV testing utilization in the context of rapid scale-up of HIV/AIDS services in rural Mozambique. Health Place 28:133-41.

Zulu LC, Kalipeni E, Johannes E, 2014. Analyzing spatial clustering and the spatiotemporal nature and trends of HIV/AIDS prevalence using GIS: the case of Malawi, 1994-2010. BMC Infect Dis 14:285. 